03

\title{
Теплообмен при смешанной конвекции расплава соли в присутствии магнитных полей
}

\author{
(ㄱ И.А. Беляев ${ }^{1}$, Д.А. Бирюков ${ }^{1, \uparrow, ~ А . В . ~ К о т л я р ~}{ }^{2}$, Е.А. Белавина ${ }^{1}$, П.А. Сардов ${ }^{1}$, В.Г. Свиридов ${ }^{1}$ \\ ${ }^{1}$ Объединенный институт высоких температур РАН, Москва, Россия \\ ${ }^{2}$ Национальный исследовательский университет „МЭИ“, Москва, Россия \\ 『E-mail: birdir@mail.ru
}

Поступило в Редакцию 29 августа 2018г.

В окончательной редакции 26 февраля 2019г.

Принято к публикации 27 февраля 2019г.

\begin{abstract}
Рассмотрены результаты экспериментального исследования опускного течения расплавов солей в однородно обогреваемой трубе под воздействием сильного поперечного магнитного поля. Исследованы изменения коэффициентов теплоотдачи и статистических характеристик температурных пульсаций под воздействием магнитного поля. Изучены особенности перехода вязкостно-гравитационного течения в вязкостно-инерционно-гравитационное при числах Рейнольдса $\operatorname{Re}=3000-5000$ под влиянием магнитного поля (число Гартмана На $=17$ ).
\end{abstract}

DOI: 10.21883/PJTF.2019.10.47752.17507

Одним из перспективных направлений развития ядерной энергетики является создание реакторов на расплавах солей и разработка гибридных термоядерных установок $[1,2]$. Создание подобных систем сопряжено с решением множества технических и научных проблем [3]. В случае использования расплавов солей в гибридных реакторах на основе реакторов типа ТОКАМАК движение расплавов солей будет происходить в сильных магнитных полях. Непосредственное воспроизведение этих условий в научных экспериментах является слишком дорогостоящим, поэтому исследования проводятся путем численного моделирования [4] и экспериментов с использованием модельных теплоносителей [5-7]. Для воссоздания условий, близких к условиям термоядерного источника нейтронов, был использован модернизированный экспериментальный стенд РК-3; в качестве модельной жидкости был выбран 30\% раствор КОН (РК-3 - ртутный контур третьего поколения [8], изначально созданный для исследования жидкокристаллических теплоносителей).

Циркуляция раствора гидроксида калия [9] в контуре обеспечивается за счет химического центробежного насоса; пульсации давления после насоса эффективно снижаются в результате совокупности работы редуктора и системы гасителей пульсаций мембранного и воздушного типа. В итоге на входе в рабочий участок (круглая тонкостенная труба с внутренним диаметром $19 \mathrm{~mm}$ и толщиной стенки $0.5 \mathrm{~mm}$, установленная внутри электромагнита) подводится поток раствора, гидродинамическая стабилизация которого происходит на первых 60 калибрах экспериментального участка, а на последующих 40 калибрах осуществляется развитие изучаемого течения. В конце рабочего участка, в области однородного магнитного поля, установлен шарнирный сканирующий зонд (рис. 1), на кончике которого закреплена хромельалюмелевая микротермопара $(250 \mu \mathrm{m})$. На внешней по- верхности рабочего участка установлен электрический нагреватель.

С помощью шарнирного зонда выполнялось сканирование температурного поля исследуемого сечения, и на основании данных о температуре и режимных параметpax рассчитывались безразмерные локальные и затем

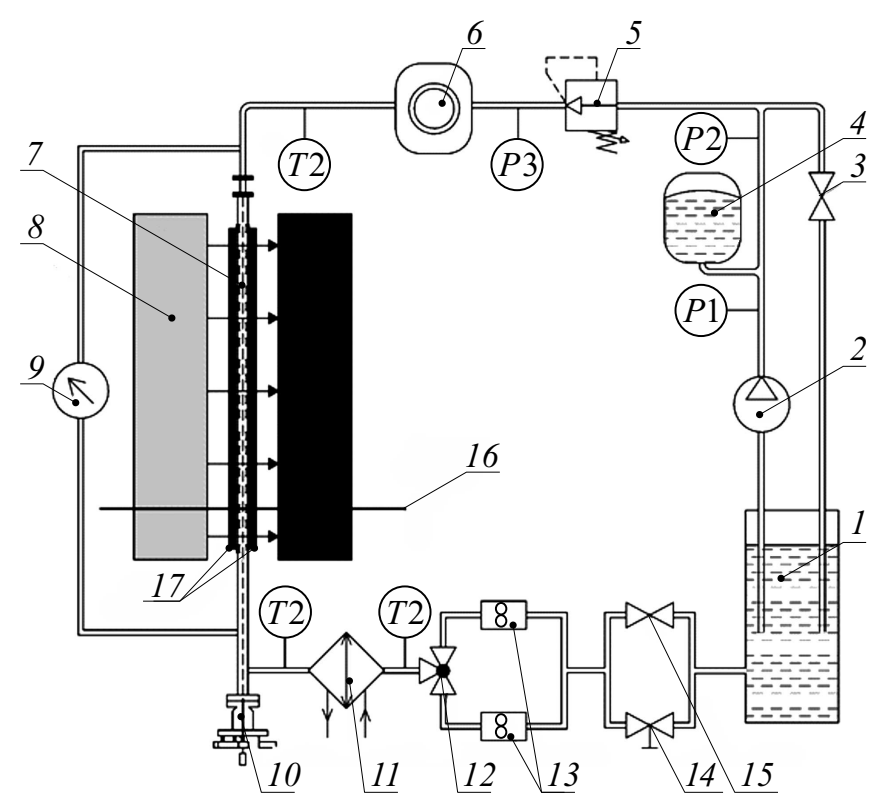

Рис. 1. Принципиальная схема модернизированного экспериментального стенда РК-3. 1 - резервуар, 2 - центробежный насос, 3 - вентиль байпасной линии, 4 - гаситель пульсаций мембранного типа, 5 - редуктор давления, 6 - гаситель пульсаций воздушного типа, 7 - рабочий участок, 8 - электромагнит, $9-$ датчик давления, $10-$ измерительный зонд, 11 - теплообменник, 12 - трехходовой шаровой кран, 13 - турбинные расходомеры, 14, 15 - вентили, 16 - исследуемое поперечное сечение, 17 - однородный обогрев рабочего участка. 


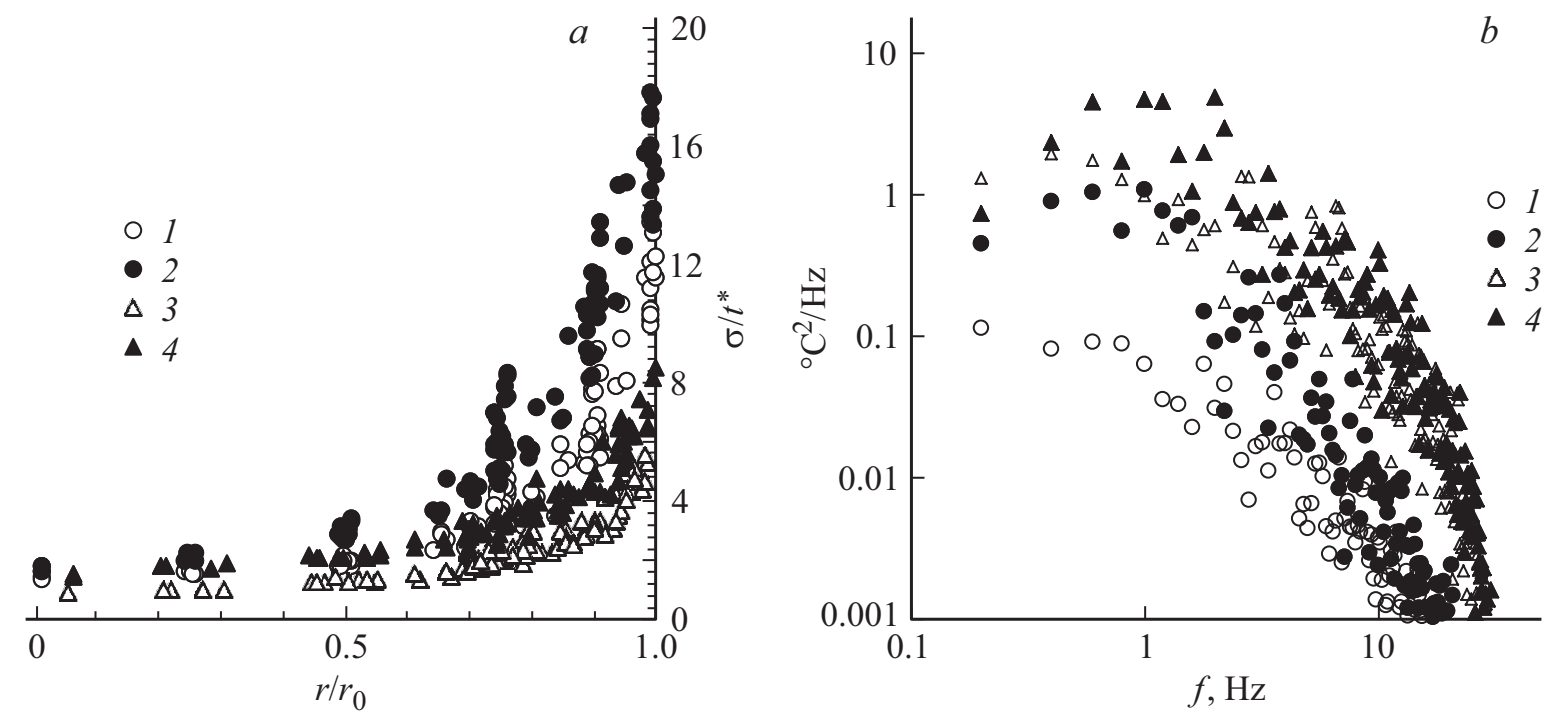

Рис. 2. $a$ - распределение безразмерной интенсивности пульсаций температуры, $\operatorname{Re}=5 \cdot 10^{3}\left(r / r_{0}-\right.$ безразмерный радиус). $b-$ спектральная плотность энергии температурных пульсаций в точке $r / r_{0}=0.9$ в зависимости от частоты $f$. $1-$ На $=0$, $\mathrm{Gr}_{A}=0.6 \cdot 10^{3} ; 2-\mathrm{Ha}=17, \mathrm{Gr}_{A}=0.6 \cdot 10^{3}, 3-\mathrm{Ha}=0, \mathrm{Gr}_{A}=4.5 \cdot 10^{3} ; 4-\mathrm{Ha}=17, \mathrm{Gr}_{A}=4.5 \cdot 10^{3}$.
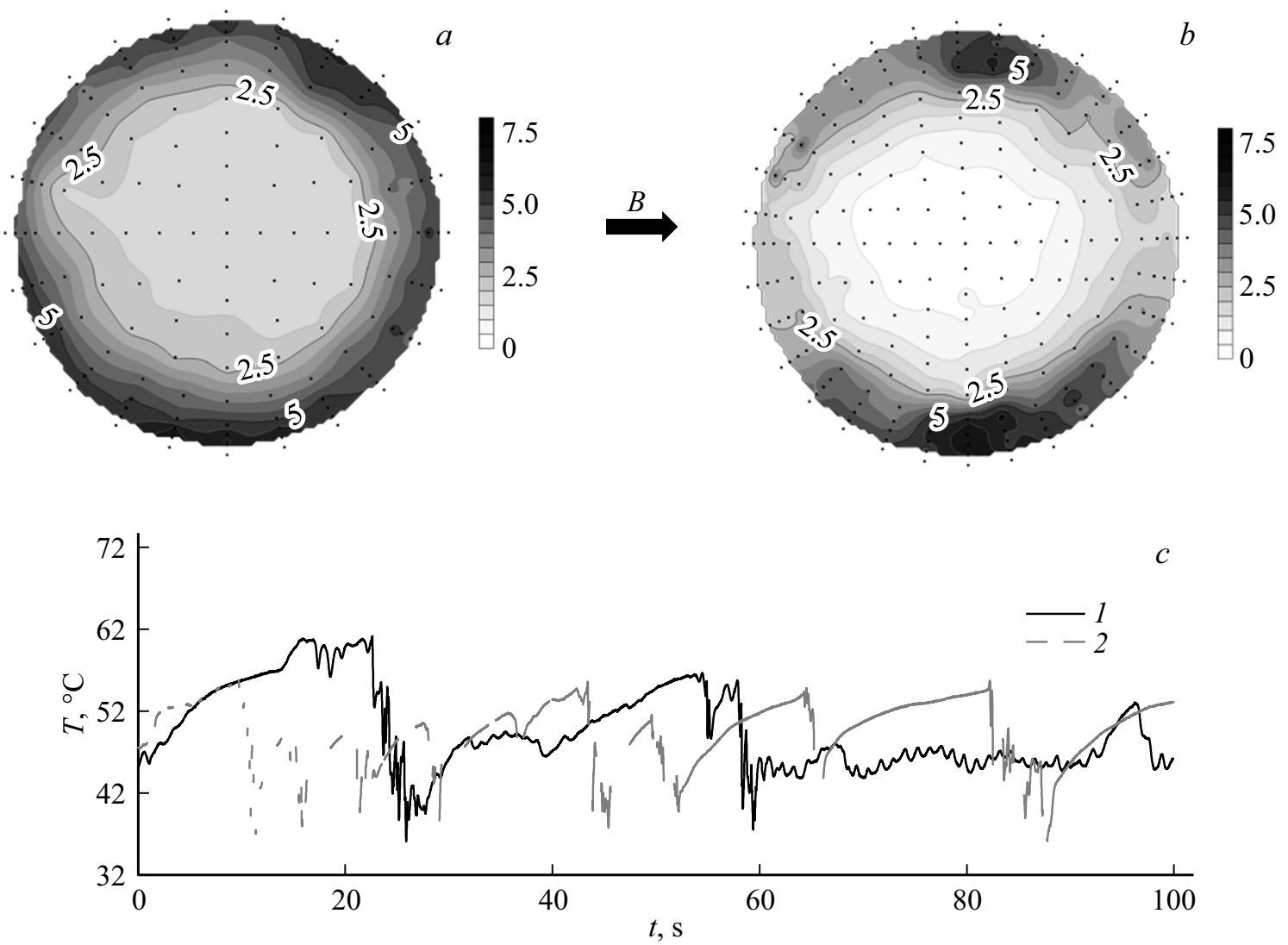

Рис. 3. $a, b-$ поля интенсивности пульсаций температуры (в сечении трубы $x / d=37, x / d-$ координата исследуемого сечения относительно диаметра трубы): $a-\mathrm{Re}=3 \cdot 10^{3}, \mathrm{Ha}=0, \mathrm{Gr}_{A}=1.1 \cdot 10^{3} ; b-\mathrm{Re}=3 \cdot 10^{3}, \mathrm{Ha}=17, \mathrm{Gr}_{A}=1.1 \cdot 10^{3} . c-$ примеры осциллограмм температуры вблизи стенки: 1 - для режима, изображенного на части $a, 2-$ для режима, изображенного на части $b$. 
средние по периметру коэффициенты теплоотдачи и числа Нуссельта. Безразмерные критерии, характеризующие режим течения, брались по аналогии с подходом, предложенным в [10]: $\mathrm{Ra}_{A}$ - аксиальное число Рэлея, $\mathrm{Ra}_{A}=\operatorname{Pr} \cdot \mathrm{Gr}_{A}$, где $\operatorname{Pr}$ - число Прандтля,

$$
\mathrm{Gr}_{A}=\frac{g \beta \delta^{4} A}{v^{2}}
$$

$\mathrm{Gr}_{A}$ - число Грасгофа, определяется по продольному градиенту среднемассовой температуры жидкости, $g$ ускорение свободного падения, $\beta$ - коэффициент объемного расширения, $\delta$ - диаметр трубы, $A-$ градиент среднемассовой температуры, $v-$ кинематический коэффициент вязкости. Аксиальные параметры вводятся для удобства сопоставления величин.

Изменения в структуре потока под воздействием магнитного поля изучались при помощи анализа интенсивности температурных пульсаций $(\sigma)$, которые обезразмеривались по методике [11] нормировкой на значение динамической температуры $\left(t^{*}\right)$. Динамическая температура получается путем вычисления через динамическую скорость

$$
t^{*}=\frac{q_{c}}{\rho C_{p} \sqrt{\frac{\tau c}{\rho}}},
$$

где $q_{c}$ - плотность теплового потока на смоченной стенке, $\rho-$ плотность, $C_{p}-$ изобарная теплоемкость, $\tau$ - напряжение трения, $c$ - коэффициент сопротивления трения.

На установке были исследованы числа Рейнольдса $(\mathrm{Re})$ до $14 \cdot 10^{3}$, Грасгофа $\left(\mathrm{Gr}_{A}\right)$ до $6.1 \cdot 10^{3}$, Гартмана (На) до 17.

Под воздействием магнитного поля величиной до $2.7 \mathrm{~T}$ (что соответствует числам Гартмана до 17) не наблюдалось существенного изменения коэффициентов теплоотдачи с учетом неопределенности экспериментальных данных. Структура турбулентных пульсаций температуры в этих режимах также не показала существенных изменений. Наиболее значимое изменение показано на рис. 2, $a$, где интенсивность пульсаций температуры увеличивается под воздействием магнитного поля. Представляет интерес тот факт, что под воздействием магнитного поля изотермические пульсации скорости должны подавляться, а значит, ожидаемо подавление пульсаций температуры. Увеличение интенсивности пульсаций температуры происходит в большей степени в области низких частот (рис. $2, b$ ) и может объясняться перераспределением энергии по спектру ввиду изменения баланса сил плавучести и вязкости под действием электромагнитных сил.

Отдельно были рассмотрены режимы с достаточно низкими числами Рейнольдса: $(3-5) \cdot 10^{3}$. В этом случае была обнаружена сложная картина течения, обусловленная переходом от вязкостно-гравитационного режима течения к вязкостно-инерционно-гравитационному, сопровождающимся образованием периодических „отрывов“ перегретых слоев жидкости от обогреваемых стенок.
В этом случае заметно и качественное, и количественное влияние магнитного поля (рис. 3 ).

Магнитное поле стабилизирует течение вблизи гартмановских пристеночных слоев, и отрывной характер течения имеет место только вблизи стенок, параллельных магнитному полю (также называемых слоями Робертса). Наблюдаемое изменение поля интенсивности пульсации температуры структурно схоже с изменениями, полученными в жидких металлах [12]. Следует отметить, что увеличение тепловой нагрузки приводит к турбулизации потока и наблюдаются стохастические пульсации температуры без периодических выбросов.

Таким образом, проведены экспериментальные исследования особенностей теплообмена имитатора расплава соли при опускном течении в вертикальной трубе при числах Рейнольдса $\mathrm{Re}=3 \cdot 10^{3}-14 \cdot 10^{3}$ под влиянием магнитного поля $(\mathrm{Ha}=17)$. В случае развитого турбулентного течения воздействие магнитного поля на структуру течения и теплообмен мало. При относительно малых тепловых потоках $\left(\mathrm{Gr}_{A} \sim 10^{3}\right)$ и малых расходах $\left(\operatorname{Re}=(3-5) \cdot 10^{3}\right)$ наблюдается сложное течение с периодическими отрывами. В этом случае обнаруживается качественное и количественное влияние магнитного поля.

\section{Финансирование работы}

Работа выполнена при поддержке Российского научного фонда (проект № 17-19-01745). Эксперименты проведены на УНУ „Ртутный стенд“.

\section{Список литературы}

[1] Азизов Э., Гладуш Г., Лопаткин А., Лукасевич И. // Атомная энергия. 2011. Т. 110. В 2. С. 84-88.

[2] Велихов Е.П., Ковальчук М.В., Ильгисонис В.И., Игнатьев В.В., Цибульский В.Ф., Андрианова Е.А., Бландинский В.Ю. // Вопр. атомной науки и техники. Сер. Термоядерный синтез. 2017. Т. 40. № 4. С. 5-13.

[3] Serp J., Allibert M., Benes O., Delpech S., Feynberg O., Ghetta V., Heuer D., Holcomb D., Ignatiev V., Kloosterman J., Luzzi L., Merle-Lucotte E., Uhlir J., Yoshioka R., Zhimin D. // Progr. Nucl. Energy. 2014. V. 77. P. 308-319.

[4] Structural materials for generation IV nuclear reactors / Ed. P. Yvon. Woodhead Publ., 2016. 684 p.

[5] Takeuchi J., Satake S., Morley N.B., Kunugi T., Yokomine T., Abdou M.A. // Fusion Eng. Design. 2008. V. 83. N 7-9. P. 1082-1086.

[6] Takeuchi J., Satake S., Miraghaie R., Yuki K., Yokomine T., Kunugi T., Morley N.B., Abdou M.A. // Fusion Eng. Design. 2006. V. 81. N 1-7. P. 601-606.

[7] Nakaharai H., Takeuchi J., Yokomine T., Kunugi T., Satake S., Morley N.B., Abdou M.A. // Exp. Therm. Fluid Sci. 2007. V. 32. N 1. P. 23-28.

[8] Belyaev I.A., Sviridov V.G., Batenin V.M., Biryukov D.A., Nikitina I.S., Manchkha S.P., Pyatnitskaya N.Yu., Razuvanov N.G., Sviridov E.V. // Therm. Eng. 2017. V. 64. N 11. P. 841-848. 
[9] Gilliam R.J., Graydon J.W., Kirk D.W., Thorpe S.J. // Int. J. Hydrogen Energy. 2007. V. 32. N 3. P. 359-364.

[10] Belyaev I.A., Zakharova O.D., Krasnoshchekova T.E., Sviridov V.G., Sukomel L.A. // Therm. Eng. 2016. V. 63. N 3. P. 214-221.

[11] Petukhov B.S., Polyakov A.F., Launder B.E. // Heat transfer in turbulent mixed convection. N.Y., 1988. 216 p.

[12] Belyaev I., Frick P., Razuvanov N., Sviridov E., Sviridov V. // Int. J. Heat and Mass Transfer. 2018. V. 127. Pt 1. P. 566-572. 